\title{
LOGARITHMS IN MULTIPLIER ALGEBRAS
}

\author{
by G. V. WOOD \\ (Received 6th February 1978)
}

\section{Introduction}

In (3) it is shown that, for a locally compact abelian group $G$ and $x \in G, \delta_{x}$ has a logarithm in $M(G)$ if and only if $x$ has finite order. Since $M(G)$ can be identified with the multipliers of $L^{\prime}(G)$, one might expect a similar result for the algebras of multipliers on $L^{p}(G)$ for $1<p<\infty$. However, in contrast, it is shown in (2) that for a locally compact abelian group $G$ and $1<p<\infty$, every translation operator on $L^{p}(G)$ has a logarithm in the multiplier algebra. Here we consider whether the same results are true for non-abelian groups.

The result for $1<p<\infty$ is easily generalised to all locally compact groups - given a deep result of Herz, (see Section 3). However, the case of $M(G)$ is not as straightforward. In fact, we can only prove the result for compact Lie groups and this is given in the next section.

For a locally compact group $G, M(G)$ will denote the measure algebra, $l_{1}(G)$ the subalgebra of $M(G)$ consisting of the discrete measures, and $M^{p}(G)$ the algebra of multipliers on $L^{p}(G)$.

\section{Measure algebras}

We aim to generalise the following result of Gillespie and West:

Theorem A ((3), Lemma 12). If $G$ is a locally compact abelian group, then $\delta_{x}$ has $a$ logarithm in $M(G)$ if and only if $x$ has finite order.

The following two observations are true for arbitrary locally compact groups.

Lemma 1. If $\delta_{x}$ has a logarithm in $M(G)$, it has one in $l_{1}(G)$. (c.f. (3), Lemma 11.)

Lemma 2. If $\mu=\Sigma \alpha(y) \delta_{y}$ is a logarithm for $\delta_{x}$ in $l_{1}(G)$, then, if $\alpha(y) \neq 0, y$ commutes with some power of $x$.

Proof. Since $\exp \mu=\delta_{x}, \mu$ commutes with $\delta_{x}$. Therefore

$$
\mu=\delta_{x^{-1}} * \mu^{*} \delta_{x}=\sum \alpha(y) \delta_{x^{-1} y x}=\sum \alpha\left(x y x^{-1}\right) \delta_{y},
$$

and so $\alpha\left(x y x^{-1}\right)=\alpha(y)$ all $y \in G$.

Now if $\alpha(y) \neq 0$, the set $\{z: \alpha(z)=\alpha(y)\}$ is finite, since $\mu \in l_{1}(G)$, and the map $z \mapsto x z x^{-1}$ is a permutation of it. In particular $x^{n} y x^{-n}=y$ for some $n$. 
To prove the main result we need the fact that for a compact Lie group $G$, the intersection of its centre $Z(G)$ and its commutator subgroup $G^{\prime}$ contains only elements of finite order. If $G$ is connected, then $Z(G) \cap G^{1}$ is a finite group. This follows from the structure theorem that $G$ is isomorphic to $\left(Z \times T_{1} \times \cdots \times T_{n}\right) / K$ where $Z$ is the identity component of the centre of $G$, each $T_{i}$ is a simple normal subgroup of $G$ (i.e. contains no connected proper normal subgroups) and $K$ is a finite central subgroup of the product, (see (1) p. 30). It seems likely that $Z(G) \cap G^{1}$ is finite for any compact Lie group $G$, since $G$ is a finite extension of its connected component. However, it is easy to see that $Z(G) \cap G^{1}$ is a torsion group, which is all that is required here.

We have the following for any compact group.

Lemma 3. If $G$ is a compact group and $x \in Z(G) \cap G^{\prime}$ then $\pi x$ has finite order for every irreducible representation $\pi$ of $G$.

Proof. We can assume that $\pi$ is a unitary representation. Let $n=\operatorname{dim} \pi$. Since $x \in Z(G)$, by Schur's lemma, $\pi x=\lambda I_{n}$ for some $\lambda \in C,|\lambda|=1$. Since $x \in G^{\prime}, \pi x \in$ $S U(n)$ - the commutator subgroup of $U(n)$. Hence $\lambda^{n}=1$ and $\pi x$ has finite order.

Proposition 4. If $G$ is a compact Lie group, $Z(G) \cap G^{1}$ contains only elements of finite order.

Proof. Suppose that $x \in Z(G) \cap G^{1}$ and has infinite order. We may assume that $x$ belongs to the connected component of $G$, since some power of $x$ must. Let $H$ be the closed subgroup of $G$ generated by $x$. Then $H$ is a monothetic group which is contained in a torus - the maximal torus containing $x$. Hence $H$ is isomorphic to $F \times T$ for a finite group $F$ and a torus $T$. In particular there is a one-dimensional character $\phi$ on $H$ such that $\phi(x)$ is not a root of unity. This representation of $H$ can be extended to an irreducible representation $\pi$ of $G(5$, vol. II $(27.46))$. Thus $\pi x$ has infinite order which contradicts Lemma 3.

The conclusion of this Proposition is true in any group where the monothetic subgroups have characters of infinite order. This is not the case in disconnected groups, e.g. the $p$-adic integers, (see (5), vol. I (24.26)).

Amongst the groups where $Z(G) \cap G^{1}$ contains elements of infinite order are the following:

Let $R$ be a commutative ring with identity 1 and characteristic zero and $G$ the group of matrices

$$
\left\{\left(\begin{array}{lll}
1 & x & z \\
0 & 1 & y \\
0 & 0 & 1
\end{array}\right): x, y, z \in R\right\} . \quad \text { Then } Z(G)=G^{1}=\left\{\left(\begin{array}{ccc}
1 & 0 & z \\
0 & 1 & 0 \\
0 & 0 & 1
\end{array}\right): z \in R\right\}
$$

Even when $R=Z$ or $R$, it is not known whether elements of infinite order have logarithms in $M(G)$. If $R=p$-adic integers, then $G$ is a compact group and the question is still open.

We can now prove the main theorem. 
Theorem 5. Let $G$ be a compact Lie group. Then $\delta_{x}$ has a logarithm in $M(G)$ if and only if $x$ has finite order.

Proof. If $x$ has finite order, then $\delta_{x}$ has a logarithm since its spectrum is only a finite set of points. Conversely, suppose $x$ has infinite order and that $\delta_{x}$ has a logarithm in $M(G)$. By Lemma 1 , we may assume that the logarithm is in $l_{1}(G)$. As in Proposition 4, we may assume that $x$ belongs to the connected component of $G$ and that the group generated by $x$ is of the form $F \times T$. Thus by taking another power of $x$ if necessary, we may assume that $x$ generates a torus. Now all powers of $x$ generate the same torus, so, by Lemma 2, we have that any $y$ in the support of the logarithm of $x$ commutes with $x$. We can thus replace $G$ by the centraliser of $x$, and hence assume that $x \in Z(G)$. Let $\sigma: G \rightarrow G / G^{1}$ be the canonical projection. Then $\delta_{\sigma x}$ has a logarithm in $M\left(G / G^{1}\right)$. By the abelian result (Theorem A) $\sigma x$ has finite order $-n$ say. Hence $x^{n} \in G^{l} \cap Z(G)$, which contradicts Proposition 4, and the fact that $x$ has infinite order. Thus $\delta_{x}$ cannot have a logarithm, and the proof is complete.

In the abelian case, whether $\delta_{x}$ has a logarithm in $M(G)$ is equivalent to whether $\delta_{x}$ belongs to the connected component of the group of invertible measures. In the non-abelian case this is not so, since the set of exponentials need not form a group. If $M(G)^{-1}$ denotes the group of invertible measures in $M(G)$ and $M(G)_{0}^{-1}$ its connected component, it is clear that $\delta_{x}$ may belong to $M(G)_{0}^{-1}$ even when $x$ has infinite order-e.g. when it is the product of elements of finite order. In the abelian case, the Arens-Royden theorem shows that $M(G)^{-1} / M(G)_{0}^{-1}$ is isomorphic to the first Cech cohomology group $H^{1}(\Delta)$ of the maximal ideal space $\Delta$ of $M(G)$. Also J. L. Taylor has characterised elements of $M(G)^{-1}$ in (6), p. 78. These results together with Gillespie and West's result prove the following.

Theorem 6. Let $G$ be a locally compact abelian group. The following are equivalent:

(1) $G$ is a torsion group;

(2) every invertible measure on $G$ has a logarithm;

(3) $M(G)^{-1}$ is connected;

(4) $H^{1}(\Delta)$ is trivial.

(In proving $(1) \Rightarrow(2)$, we use (6), Theorem 8.2.4 and the fact that the dual group of a torsion group is totally disconnected and hence has its first Céch cohomology group trivial.)

Thus we have two natural conjectures for non-abelian groups.

Conjecture 1. $G$ is generated by its torsion elements if and only if $M(G)^{-1}$ is connected.

Conjecture 2. $G \cap M(G)_{0}^{-1}$ is the subgroup of $G$ generated by the torsion elements of $G$. 


\section{Multiplier algebras}

In (2), Gillespie proves:

Theorem B. (2, Theorem 2) For a locally compact abelian group $G$ and $1<p<\infty$, every translation operator $L_{x}$ in $M^{D}(G)$ has a logarithm.

This result can be extended to all locally compact groups using a result of Herz-(4), Theorem A-which shows that, if $H$ is an amenable subgroup of $G$, there is a natural embedding of $M^{p}(H)$ in $M^{p}(G)$. Thus, taking $H$ to be an abelian subgroup of $G$ containing $x, L_{x}$ has a logarithm in $M_{p}(H)$ which has a natural extension in $M_{p}(G)$. Since the embedding is an algebra map and preserves $L_{x}$, the result is proved. Thus we have, for $1<p<\infty$.

Theorem 7. Let $G$ be a locally compact group and $M^{p}(G)$ the algebra of multipliers on $L^{p}(G)$. Then the translation operators in $M^{p}(G)$ have logarithms in $M^{p}(G)$.

However there are many open questions in connection with the connected component of the group of invertible multipliers. For example, even in the abelian case, it seems to be unknown whether every invertible multiplier in $M_{p}(G)(1<p<\infty)$ has a logarithm.

\section{REFERENCES}

(1) BREDON, Introduction to compact transformation groups (Academic Press, 1972).

(2) T. A. Gillespie, Logarithms of $L^{p}$ translations, Indiana Univ. M. J. 24 (1975), 1037-1045.

(3) T. A. GILlESPIE, and T. T. WEST, Weakly compact groups of operators, Proc. Amer. Math. Soc. 49 (1975), 78-82.

(4) C. HERZ, Harmonic synthesis for subgroups, Ann. Inst. Fourier 23, 3 (1973), 91-123.

(5) HEwITT and Ross, Abstract Harmonic Analysis, vol. I and II, (Springer-Verlag, 1963 and 1970).

(6) J. L. TAYLOR, Measure algebras, CBMS No. 16, (1973).

Department of Pure Mathematics

UNIVERSITY COLLEGE OF SWANSEA

SINGLETON PARK

SWANSEA, SA2 8PP 